\title{
Corela
}

Cognition, représentation, langage

HS-14 | 2013

Calcul du sens et contexte

\section{Sous-minimalité, planification et effets de contexte sur la représentation sémantique}

\section{Nick Riemer}

\section{OpenEdition}

\section{Journals}

Édition électronique

URL : http://journals.openedition.org/corela/3424

DOI : $10.4000 /$ corela.3424

ISSN : $1638-573 \mathrm{X}$

\section{Éditeur}

Cercle linguistique du Centre et de I'Ouest - CerLICO

Référence électronique

Nick Riemer, "Sous-minimalité, planification et effets de contexte sur la représentation sémantique », Corela [En ligne], HS-14 | 2013, mis en ligne le 24 juin 2014, consulté le 02 mai 2019. URL : http:// journals.openedition.org/corela/3424 ; DOI : 10.4000/corela.3424

Ce document a été généré automatiquement le 2 mai 2019.

\section{(c) (i) (2)(2)}

Corela - cognition, représentation, langage est mis à disposition selon les termes de la licence Creative Commons Attribution - Pas d'Utilisation Commerciale - Partage dans les Mêmes Conditions 4.0 International. 


\title{
Sous-minimalité, planification et effets de contexte sur la représentation sémantique
}

\author{
Nick Riemer
}

\section{Introduction}

$1 \quad$ Le bref article qui suit propose des réflexions générales et spéculatives sur les rapports entre le sens et le contexte, du point de vue disciplinaire de la sémantique lexicale. Inutile d'insister à quel point les questions de contexte sont capitales pour la linguistique en générale et pour la sémantique en particulier. Si on prend la notion de sens dans son acception traditionnelle, selon laquelle le sens d'un lexème est l'ensemble de facteurs non-syntaxiques et non-pragmatiques qui déterminent les capacités référentielles et inférentielles des expressions (voir par ex. Rastier 2001, p. 82ff; Nyckees 1998), le contexte devient une partie incontournable de la sémantique: le sens, c'est ce qui détermine l'usage référentiel/inférentiel - qui, lui, se produit forcément toujours dans un contexte plus large. Il est donc naturel de s'attendre à ce que les rapports sens-contexte se trouvent au centre des réflexions sémantiques aussi bien que pragmatiques.

2 Toutefois, l'hypothèse de base de toute sémantique linguistique permet très souvent d'ignorer l'influence du contexte sur la représentation sémantique. Selon cette hypothèse, toute expression lexicale comprend un noyau invariant de sens - le sens littéral - toujours présent lors de l'énonciation. Cette conception du sens a pour conséquence de marginaliser le rôle du contexte, en déclarant une zone de sémantique pure, affranchie de tout apport contextuel. Cela entraîne un certain isolement de la sémantique, à la fois, au sein de la linguistique, et des autres sciences cognitives. La sémantique linguistique ne ferait donc que bénéficier des liens plus étroits avec tout autre secteur de la linguistique ou des disciplines connexes (philosophie, psychologie, sciences cognitives...) qui poursuivent des recherches sur les problèmes de contexte. Impossible de récolter les fruits de ces disciplines connexes sans franchir un écart 
disciplinaire assez important, démarche qui entraîne évidemment des risques. Dans cet article je n'ai pas hésité à mettre à contribution des travaux en pragmatique, ainsi que certains résultats obtenus dans la recherche sur le traitement sémantique et la cognition en général, dans l'espoir qu'une perspective disciplinaire fédératrice aura une chance d'apporter des éclaircissements quant à la question des rapports entre sens et le contexte.

\section{La position standard : les représentations riches en informations et le caractère " supplétif » du contexte}

3 En analysant les rapports entre le sens et l'usage, la théorie linguistique traditionnelle, que ce soit d'inspiration structuraliste, cognitiviste, logico-formelle, ou bien lexicographique, a minimisé l'importance du contexte. Selon la conception la plus courante, le contexte ne fait que fournir des élément de sens qui manquent dans la signification littérale. Voilà une conception très répandue, auxquelles seulement les théories pragmatiques «contextualistes » ou bien « radicales" dérogent (voir Breheny 2002). Selon cette tendance de la pragmatique, le contenu vériconditionnel de la proposition communiquée est déterminée, au moins en partie, pragmatiquement. Pourtant, ce cadre de recherche n'a quasiment pas du tout influé sur la sémantique lexicale, qui continue à voir dans le contenu sémantique codé la source principale du sens véhiculé dans les actes de communication (voir par ex. Geeraerts 2010).

Pour mieux analyser comment les rapports sens-contexte sont traités par la plupart de sémanticiens lexicaux, il faut prendre en compte les divers facteurs qui, selon la conception la plus habituelle, entrent en jeu dans la compréhension d'un énoncé, en commençant l'analyse du côté de l'auditeur. Avant l'énonciation d'une proposition, celuici se trouve dans un certain état cognitif initial, se représentant diverses assomptions, croyances, etc. En idéalisant de manière assez brutale, on va considérer cet état informationnel initial comme la représentation d'un ensemble de propositions (cf. les « assumptions » de la théorie de la pertinence). Après l'articulation de l'énoncé par le locuteur, la compréhension chez l'auditeur se passe en deux temps. Tout d'abord, on se représente toute l'étendue des informations linguistiques (c.-à-d., codées) véhiculées par l'énoncé. Ensuite, on fait appliquer à ces informations codées les propositions de l'état informationnel initial de sorte que la proposition communiquée par l'énoncé soit dérivée. Selon cette conception, on ne peut plus répandue en sémantique et pragmatique, le contexte entre en jeu en tant que source des propositions de l'état informationnel initial pour compléter, de manière "supplétive ", le sens strictement sémantique. Par exemple, l'énoncé il pleut livre la proposition dérivée il pleut en ce moment dans ce lieu, l'auditeur opérant un procédé d'enrichissement pragmatique en faisant appel à sa connaissance préalable de l'ancrage spatio-temporel du contexte, partie de son état informationnel initial, pour compléter l'interprétation. De pareille façon, l'énoncé il faut commencer, prononcé au début d'une partie de ping-pong, recevra l'interprétation il faut maintenant commencer à jouer au ping-pong, non pas jouer au tennis. L'apport du contexte permet à l'auditeur de dériver une proposition à partir de l'énoncé en complétant les informations purement sémantiques.

5 Ce caractère "supplétif» du composant pragmatique est entraîné par la manière strictement hiérarchisée dont on aborde la communication au sein des modèles standard. On peut résumer cette hiérarchisation de la manière suivante, en forme du principe 
REOCS («Représentation Entière Obligatoire du Contenu Sémantique»), que l'on va articuler en deux parties :

1. Pour tout énoncé $\mathrm{E}$, l'auditeur $\mathrm{A}$ comprend $\mathrm{E}$ si et seulement si le contenu littéral sémantique entier de chaque expression de $\mathrm{E}$ est représenté (dans la mémoire de travail). Autrement dit, tout le sens littéral de E est obligatoirement représenté par A pendant la compréhension.

2. Pour tout $\mathrm{E}$, les informations d'ordre pragmatique n'entrent en jeu que pour suppléer soit les déficits informationnels, soit la défaillance du contenu sémantique littéral.

6 Regardons de plus près la deuxième partie. Le cas le plus évident d'un déficit informationnel dans le contenu sémantique littéral est la référence. L'identification des référents, condition capitale pour la dérivation de la proposition communiquée, relève de la pragmatique, non pas de la sémantique. En plus de l'identification des référents, Recanati (2012) reconnaît deux autres procédés qui fournissent les informations qui manquent dans le contenu sémantique : " précisation » (« specifization ») et « construction de sens » (« sense construction»).

7 Quant à la défaillance du contenu sémantique littéral, on l'observe dans les phénomènes comme la métaphore, l'ironie, l'implicature conversationnelle etc. Dans de tels cas, l'auditeur rejette ou bien est obligé d'aller au-delà de la proposition littéralement communiquée parce que celle-ci ne correspond manifestement pas avec l'intention du locuteur.

8 Voilà donc une conception traditionnelle du rapport sémantique-pragmatique dans l'interprétation des énoncés. Selon cette conception, les informations pragmatiques contextuelles sont supplétives par rapport au niveau sémantique de base, celui des informations codées représentées dans la mémoire de travail de l'auditeur pendant la compréhension d'un énoncé (cf. la notion de "focal attention» : Givón 2001) . Même dans l'approche contextualiste on fait l'hypothèse de la REOCS, postulat qui rend le contexte supplétif. Selon cette théorie, le sens littéral ne suffit pas pour déterminer le contenu propositionnel entier, mais il est pour autant représenté pleinement pendant la compréhension des énoncés.

9 La robustesse de ce modèle dans la sémantique lexicale cadre mal et avec les progrès récents de la recherche pragmatique, et avec l'exploration des processus cognitifs en général, ainsi que celle du traitement sémantique des énoncés en particulier. Dans tous ces domaines, la recherche contemporaine a de plus en plus tendance à remettre en question le rôle du sens littéral comme fondement du processus interprétatif. Je suggérerai plus bas (\$3) que la première partie du principe REOCS - la représentation entière obligatoire de tout le contenu sémantique d'un lexème - est erronée. Avant cela, on peut constater que la deuxième partie, selon laquelle l'enrichissement pragmatique n'a lieu qu' après que l'interprétation littérale a été résolue, est parfois remise en question par l'étude de la métaphore. Selon la conception direct access, les auditeurs accèdent directement à l'interprétation métaphorique d'un énoncé, sans être préalablement obligés de considérer, puis rejeter, le sens littéral, même si ce dernier demeure néanmoins disponible (Gibbs 2002; voir pourtant Giora 2012). Dans ce qui suit, je vais suggérer que cette conception n'est aucunement limitée à la métaphore, et je développerai un raisonnement similaire pour des énoncés non-figuratifs, en remettant en question l'importance de l'information sémantiquement codée, et en soulignant le rôle des facteurs contextuels dans la compréhension en temps réel. 


\section{Contexte et sous-minimalité des représentations sémantiques}

10 Si le rôle de la pragmatique est strictement "supplétif», de quelle nature est le sens littéral? Selon la réponse habituelle dans la sémantique lexicale, les significations échangées entre le locuteur et l'auditeur sont des représentations mentales complètes, dans le sens qu'elles comprennent toute l'information factuelle qui, de manière invariable, caractérise le référent et le distingue d'autres référents possibles, quelle que soit la pertinence de cette information dans le contexte de discours actuel (voir par ex. Langacker 1987 : 373; Cann, Kempson et Gregoromichelaki 2009; Wierzbicka 1996). La conceptualisation à laquelle correspond un sens

«embodies [the] commonality [between different referents of the term], while excluding the many properties that vary from one instance to the next» (Langacker 1987 : 373).

11 On pourrait par exemple analyser dans ce cadre le sens du lexème arbre comme un schéma conceptuel présentant " a tall plant with branches, leaves and bark» (Langacker 1987 : 373). Cette information constitue le noyau central (selon certaines approches, le prototype) du sens du mot arbre, qui, lui, subira des variations importantes dans des contextes différents.

12 Malgré l'évidence apparente de cette analyse, il est évidemment bien possible qu'un locuteur fasse référence à un arbre sans feuilles, voire, dans certaines circonstances bien précises - par exemple quand l'arbre en question se trouve dans une scierie - sans branches ni écorce. Pour autant, on suppose couramment que les échanges linguistiques dans lequel figure le mot arbre observent le principe REOCS. On fait l'hypothèse que le contenu littéral sémantique entier est réellement présent sur le plan psychologique/ cognitif, bien qu'il arrive que certains composants de cette conceptualisation soient mis en relief («profiled ») et d'autres mis en arrière-plan («backgrounded »: Langacker 1987) selon le contexte de discours actuel.

Cette conception du rôle du sens littéral devient de plus en plus douteuse, grâce aux recherches dans la psychologie (Barsalou 1999, 2008), les sciences cognitives (Clark 1997, 1998, Van Gelder 1998, Beer 2000, de Fornel et Quéré 1999, etc.), l'intelligence artificielle (Brooks 1991), ainsi que dans certains courants de la linguistique même (Pinker and Prince 1988 ; Hopper 1998), y compris la phonologie (Port 2010, Port et Leary 2005). Ce qui en ressort, c'est que l'on reconnaît de plus en plus les avantages d'un cadre théorique dans lequel la place des connaissances conceptuelles détaillées et élaborées se voit restreinte. De tels travaux se poursuivent maintenant depuis plus d'une décennie. Néanmoins, et comme c'est souvent le cas, la linguistique générale tarde à en rendre compte, malgré des tentatives ici et là (théorie de la pertinence, certains courants de la linguistique cognitive...). Le nouveau paradigme dont il est question souligne que les analyses traditionnelles des processus mentaux «focus too much on the passive storage of information and too little on the importance of situated action" (Barsalou 2008:623; cf. Glenberg 1997).

Dans le présent article, nous allons nous pencher sur une alternative fondamentale au postulat des représentations sémantiques complexes du type classique comme les moyens dont le système cognitif gère l'action langagière. 

que le système cognitif, au lieu de traiter les représentations mentales, exploite souvent les propriétés de l'environnement externe pour diriger le comportement intelligent de l'organisme (Gibson 1986 [1979], Kirsh 1995, Brooks 1991, Anderson 2003). Les illustrations de cette possibilité sont abondantes dans les domaines non-linguistiques ; en revanche, le langage paraît traditionnellement le domaine le moins propice à ce genre d'analyse. Néanmoins, considérons la manière dont on pourrait faire valoir cette démarche explicative dans les questions sémantiques (voir Riemer 2013 pour plus de détails). vide, ou bien dans un parc. Sur la table il y a une seule raquette. Une des personnes demande à l'autre de "passer la raquette". Selon les modèles ordinaires de la compréhension des énoncés, le sens de raquette recevrait un traitement exhaustif, la représentation sémantique de ce mot entier étant activée pour permettre à l'auditeur de déterminer les aspects de l'environnement actuel qui la satisfont. L'auditeur accèderait donc aux informations contenues dans cette représentation, et essaierait de les réconcilier avec le contexte de discours actuel. La raquette sur la table satisfaisant les paramètres du sens du mot raquette, l'auditeur peut identifier le même référent que le locuteur, en vérifiant que la raquette devant lui possède les propriétés requises par la définition du mot raquette, à savoir « objet à surface plate tenu dans la main pour frapper les balles ".

17 Pourtant, dans le contexte actuel, aucun élément de cette définition n'est pertinent. L'auditeur n'est aucunement dans l'obligation de se rendre compte des propriétés de la raquette telles que la définition les précise ; il lui faut tout simplement reconnaître que la raquette est le seul objet devant lui que l'on pourrait passer. En supposant que le locuteur observe les principes coopératifs ordinaires, l'auditeur n'est aucunement dans l'obligation de représenter les détails qui figurent dans une définition, même minimale, comme celle que j'ai donnée. Tous ces détails sont redondants, parce que l'auditeur n'est pas contraint de faire la différence entre la raquette et un autre objet. En rendant inutile tout appel aux informations codées, le contexte devance la sémantique codée en fournissant à l'auditeur toutes les informations nécessaires pour une interaction linguistique réussie. En maintenant l'hypothèse que la demande du locuteur est raisonnable, l'auditeur peut répondre à la requête de "passer la raquette » en passant le seul objet dans l'environnement capable d'être passé. La structure de l'environnement fait que la représentation sémantique entière du mot raquette ne doit pas être impliquée dans la réponse de l'auditeur. L'auditeur peut «comprendre» sans utiliser un seul composant de la représentation sémantique liée avec le mot raquette. Dans ce cas, le principe REOCS ne s'applique donc pas.

18 Cela n'implique aucunement que la connaissance des raquettes n'est pas disponible chez l'auditeur. L'auditeur possède évidemment une large gamme de connaissances normatives concernant les raquettes. Autrement dit, il possède le concept « raquette ». Toutefois, ce concept est redondant pour comprendre l'énoncé du locuteur, parce que le contexte ne fournit qu'une seule réponse possible à la demande.

19 Cette illustration soulève la possibilité que des facteurs contextuels peuvent déterminer quels aspects de la connaissance d'un agent cognitif sont appliqués pendant le traitement mental des énoncés. Dans l'exemple en question, plus l'environnement devient complexe, moins le contexte fournit l'information nécessaire pour une interaction réussie, obligeant 
l'auditeur à activer leur concept « raquette ». Au cas où la table de ping-pong présente une raquette et une balle, il faudra à l'auditeur représenter assez d'informations sur les deux pour faire la différence entre elles. Dans une telle situation, il faudra contraster le trait «sphérique » avec le trait " plat » pour déterminer quel objet est exigé. Au delà de ces deux traits-là, les autres informations comprises dans les définitions de raquette et de balle ne seront pas nécessaires.

Cette illustration donne à penser que le nombre de traits actifs nécessaires au sein d'une représentation sémantique pour assurer son traitement réussi peut varier selon le nombre de référents possibles. D'autres facteurs contextuels entrent en jeu aussi : par exemple, si l'auditeur croit que le locuteur fait souvent des demandes peu raisonnables, il sera amené à traiter plus profondément la demande "passer la raquette ", surtout pour déterminer s'il existe quelque chose dans le contexte actuel qui satisferait raquette. Par contre, un auditeur qui croit que le locuteur ne demande jamais que la raquette pourra se passer de l'effort de traitement cognitif du mot raquette, même si l'environnement comprend bien d'autres objets capables d'être passés. Moins la situation exige un choix, moins l'agent est dans l'obligation d'exercer un contrôle cognitif, et plus restreintes deviendront les représentations qu'il faut postuler pour rendre compte de sa réponse.

Dans de telles situations, l'auditeur « comprend » parfaitement les mots de l'énoncé, mais il n'a engagé qu'une partie négligeable de la représentation sémantique du mot « raquette ». L'auditeur n'a certainement pas représenté la définition complète de raquette: seulement une partie infime des informations factuelles représentables est pertinente, et cette partie seulement se voit utilisée.

La situation que nous venons de décrire n'est aucunement factice. Les échanges linguistiques sont toujours enracinés dans des situations précises, et axés sur des buts particuliers. Dans le discours naturel, des facteurs comme l'attention (Verschueren 1999; Sanford 2002), la pertinence (Sperber and Wilson 1995), ainsi que les biais naturels de notre orientation au monde, rendant certains aspects de l'environnement plus saillants que d'autres (Merleau-Ponty 1946; Gadamer 1960 ; Dreyfus 1991) servent à focaliser les possibilités référentielles. Comme résultat de tels facteurs, dans le cas typique, les participants dans une conversation ne sont obligés de représenter qu'une partie du contenu sémantique supposé d'une expression. Cela entraîne des conséquences importantes pour toute tentative de théoriser la représentation sémantique. Plus haut, on a souligné le fait que, selon l'interprétation habituelle, les représentations sémantiques comprennent le noyau informationnel invariable supposé toujours présent, à travers tous les contextes d'emploi différents (sous réserve d'emploi non-littéral). Voilà donc une conception minimale du sens: le sens d'une expression, selon cette conception, ne comprend que le minimum d'informations nécessaires pour assurer la différenciation entre celle-ci et les autres expressions avec lesquelles elle entretient un rapport paradigmatique.

Par souci d'économie théorique, il faut éviter de fonder les modèles des processus mentaux sur des tâches cognitives inutiles. Comme on vient de le démontrer, la compréhension peut se produire sans la présence d'une représentation sémantique minimale. Il est donc plausible de soutenir l'idée suivante: les représentations sémantiques impliquées dans l'action linguistique sont souvent sous-minimales: elles ne comprennent même pas toute l'information nécessaire pour assurer la différenciation paradigmatique des expressions, ne comprenant que les informations requises pour assurer la référence dans le contexte actuel. 
On peut voir dans la sous-minimalité une forme particulière de la sous-spécification (voir par ex. Pustejovsky 1998). Comme les autres formes de ce phénomène, sa présence dans le discours naturel s'explique comme le résultat d'un principe du moindre effort. Comme l'exprime Frisson (2009: 118), lorsqu'il s'agit des significations sous-spécifiées :

the processor does not have to make a selection between competing interpretations but can immediately proceed with the more abstract interpretation and flesh it out on the basis of further information in the text or when necessary. Obviously, this allows for much more flexible semantic processing even though the resulting online representation of words in a text might not be as detailed as is often assumed.

Il est intéressant de constater que l'on reconnait la réalité d'un phénomène tout à fait équivalent à la sous-minimalité dans la recherche sur l'acquisition de la langue maternelle. Clark (2009 : 287) nous invite à considérer la scène suivante :

Imagine a child, Anna, holding a drink container and waiting for the adult to fill it with milk. (She already knows the word cup, but not mug.) The adult then says to her, "Can you give me your mug?" At this point, Anna has only one thing to give, so she can set up in memory what she has inferred about the meaning of the new term mug for the next time she hears it...

La sous-minimalité apparaîtrait donc déjà pendant l'acquisition de la langue maternelle. En effet, sans un tel processus il est difficile de savoir comment les représentations sémantiques pourraient être remplies avec les contenus pendant cette première étape d'apprentissage. Si la sous-minimalité s'avère efficace comme moyen d'inférer les sens des mots pendant l'acquisition, on pourrait demander à quel titre on l'abandonnerait une fois atteinte la maturité, étant donné les avantages cognitifs en toute probabilité apportés par ce phénomène.

De telles considérations sont riches de conséquences pour notre analyse du rapport entre le sens et le contexte. Selon les approches contextualistes ou radicales - on l'a déjà évoqué - une partie importante du contenu d'un énoncé est dérivée pragmatiquement. En postulant la sous-minimalité, on renchérit sur cette idée : dans des cas comme celui de la table de ping-pong, le contexte rend le sens codé lui-même redondant, en fournissant des informations qui, selon l'hypothèse habituelle, constituent le contenu sémantique. Là où le principe REOCS ne s'applique pas, le contexte remplit un rôle constitutif, non pas supplétif, dans la production de sens.

Des travaux récents en psycholinguistique et en neuroscience cognitive soutiennent cette hypothèse. Une tradition longue de résultats expérimentaux confirme que le traitement sémantique est souvent superficiel, même en langue écrite, les lecteurs n'ayant pas conscience des sens littéraux des expressions dans les cas où ces derniers sont incongrus dans leurs contextes. On peut évoquer à ce titre l'illusion célèbre de Moïse (Erickson et Matteson 1981). On a posé aux locuteurs la question «Combien d'animaux de chaque espèce Moïse embarqua-t-il sur l'Arche? ». La plupart des répondants donnent la réponse " deux », alors qu'ils auraient dû répondre "aucun», Noé, et non pas Moïse, étant évidemment le personnage biblique en question. De tels résultats amènent à considérer que le traitement de sens est souvent incomplet chez les sujets expérimentaux (Smith, Shoben, and Rips 1974 ; Erickson \& Mattson, 198 ; Anderson 1983 ; van Oostendorp \& de Mul, 1990 ; van Oostendorp \& Kok, 1990 ; Barton and Sanford 1993; Sanford 2002 ; Daneman 2006). Comme l'exprime Sanford (2002 : 201-2) :

The [current] findings with respect to semantic processing suggest that a primary determinant of the degree to which the semantics of a word is recruited during 
comprehension is the fit of the word to the basic situation under discussion. If the word has a low relevance to the situation, then it will receive shallower processing. This idea assumes that it is possible to check rapidly for the fit of a word to a situation. Such a mechanism could be realised if part of semantic memory was structured in terms of words (lemmas) that tend to be used together. généralement valable :

« readers have a propensity toward partial or incomplete semantic processing of words and phrases, and they will often assume coherence as a default, as long as a satisfactory level of global coherence has been established » $(2006: 85)$.

Selon Sanford (2002), toute l'information sémantique comprise dans un lexème n'est pas forcément employée pendant la compréhension, et il existe beaucoup de cas où la pragmatique remplace la sémantique.

Un rôle «maximaliste" pour le contexte de la sorte dont il est question ici va évidemment déranger la méthodologie standard de la sémantique lexicale, axée sur la reconstruction, de façon psychologiquement plausible, des représentations sémantiques, en se rapportant aux emplois référentiels et inférentiels des expressions. Or, cette ambition se voit remise en question par la conclusion que les propriétés référentielles et inférentielles des expressions ne sont toujours pas le produit de leur sens codé. Si cette conclusion s'avère correcte, cela va infirmer l'affirmation selon laquelle les représentations sémantiques postulées en sémantique lexicale constituent bien une partie centrale des dispositifs cognitifs responsables de la référence. Pour tout chercheur désireux de dévoiler les représentations réellement employées dans la cognition langagière, un problème à la fois méthodologique et de fond vient de se poser.

\section{La structure sémantique et la variation contextuelle}

La deuxième perspective pour penser le rapport entre le sens et le contexte est le continuum de variation au sein du discours naturel.

On présente habituellement le contexte comme les conditions ou l'environnement entourant l'énoncé. Ceci fait du contexte un paramètre externe au locuteur, qui influe sur les propriétés linguistiques de l'énoncé. Quant à ces propriétés linguistiques et leur rôle dans l'interprétation, les aspects à prendre en compte se limitent à deux facteurs, l'information sémantique d'un côté, et les processus pragmatiques qui se déclenchent pendant son traitement de l'autre. Il s'agit là certainement d'une idéalisation extrême. Le cerveau tout entier est impliqué pendant les actes de parole ; en plus de leurs compétences sémantiques et pragmatiques, les locuteurs et les auditeurs retiennent évidemment pendant le traitement des énoncés toute leur structure psychologique, et des facteurs psychologiques généraux influent sur le cours du traitement des énoncés. En plus du contexte externe, on peut donc reconnaître un contexte interne ou cognitif, dans le sens d'une combinaison de facteurs cognitifs non-linguistiques qui se répercutent sur les propriétés de l'énoncé, mais qui se trouvent à l'intérieur de l'environnement cognitif du locuteur et ne sont ni sémantiques ni pragmatiques. 


\subsection{Contextes planifiés et contextes libres}

34 Ce qui frappe dans la conception reçue de la sémantique, c'est la manière dont on pose un seul genre de représentation sémantique qui serait invariable quel que soit le contexte de discours. Malgré toute variation de contexte, on fait couramment l'hypothèse que les représentations sémantiques impliquées dans le discours sont des structures conceptuelles complètes et riches en informations qui emmagasinent les connaissances du monde, dont certaines parties sont engagées de manière sélective en fonction des besoins contextuels particuliers. Cependant, il est bien connu que dans tout autre domaine de la structure linguistique, le discours naturel n'est pas homogène, mais comprend une gamme de variétés qui diffèrent l'une de l'autre en fonction, surtout, de la concentration mentale des participants (voir Schilling-Estes $2007: 174$ ). Dans ce qui suit, on va se pencher justement sur la question de la concentration du locuteur, question capitale pour l'analyse du sens.

On admet depuis Labov (1972) que la concentration consacrée par le locuteur à ses mots se répercute sur les énoncés. Plus récemment, les psycholinguistes se rendent à l'hypothèse selon laquelle en plus de la phonologie, la morphosyntaxe, ou bien le discours (voir Eckert 2000 ; Coupland 2001), la sémantique aussi est susceptible d'être touchée par ce paramètre. Selon Sanford $(2002,189)$ les objets sur lesquels on focalise l'attention reçoivent un traitement sémantique plus profond et plus important - «a common enough idea in the psychology of attention ", comme il le souligne. Cet hypothèse fait du degré de l'attention une variable contextuelle qui agit sur la représentation sémantique des énoncés. Admettons cette hypothèse, et considérons ses conséquences pour les analyses sémantiques traditionnelles.

Avant de pouvoir cerner l'influence de l'attention sur la sémantique, il faut faire la différence entre deux modalités de discours distinctes, la modalité planifiée et la modalité libre (cf. Ochs 1979). Cette distinction est proche de plusieurs distinctions bien connues dans la recherche sur la variation linguistique (Labov 1972 ; Eckert 2000 ; Coupland 2007 ; Schilling-Estes 2007). La modalité planifiée est engagée dans tout contexte où le locuteur fait attention de manière consciente aux effets communicatifs de ses propos. En raison de cette attention, le locuteur a plus conscience des contraintes normatives auxquelles son discours est soumis (Verschueren 1999). Dans le contexte contraire, les contextes libres ou non-planifiés, les participants ne prêtent pas d'attention particulière à ces facteurs; ils parlent de façon spontanée, sans surveillance métalinguistique.

L'intérêt de la distinction discours libre/discours planifié se trouve dans l'hypothèse suivante : c'est dans le discours planifié que les analyses décompositionnelles complètes des significations, telles que l'on avance habituellement dans la sémantique, ont le degré de réalité psychologique le plus grand. Dans le discours planifié, les locuteurs sont conscients de manière explicite des contraintes factuelles et communicatives auxquels leurs énoncés sont soumis, et visent de façon intentionnelle à les observer. Les structures décompositionnelles pouvant être interprétées comme des résumés de ces contraintes, on est autorisé à soutenir que les analyses sémantiques décompositionnelles peuvent dans le cas du discours planifié correspondre aux structures mentales réelles impliquées dans la planification et l'articulation des énoncés.

Il va sans le dire que les modalités libres et planifiées doivent être vues comme les deux points terminaux d'un continuum. Le discours planifié se produit sous le contrôle 
conscient, réfléchi et attentif du locuteur. Dans cette modalité, on planifie et produit les énoncés de manière très délibérée, et on fait conformer le discours le plus possible aux normes explicites. Les locuteurs sont conscients de la relation de correspondance nécessaire entre les énoncés et les référents pour que la communication soit efficace. Pour cette raison, c'est dans les contextes planifiés que le principe REOCS va le plus souvent obtenir.

Le discours planifié est susceptible surtout d'avoir lieu dans deux circonstances précises :

1. lorsque le locuteur doute que ses paroles produiront le résultat souhaité, et donc lorsqu'il est obligé de bien former ses énoncés pour les rendre plus efficaces

2. dans des situations de "panne " communicative réelle ou prévue, lorsque les choix linguistiques du locuteur ont échoué quant à leur effet souhaité, ou que le locuteur estime qu'un tel échec est susceptible de se produire.

Ces deux circonstances peuvent se produire dans tout échange conversationnel, même le plus informel, donnant lieu ainsi au discours planifié. Dès que le locuteur essaie intentionnellement de faire conformer ses mots à un but précis, on est devant un contexte planifié.

41 Le discours libre présente les propriétés contraires. Spontané, non pas planifié de manière consciente, il n'est pas l'objet d'un degré élevé d'attention métalinguistique, ni accompagné par un effort délibéré de respecter les normes publiques. Il est susceptible de se produire lorsque le locuteur estime qu'il y a peu de chances que son intention communicative soit frustrée; ne risquant pas l'échec communicatif, le locuteur est dispensé de faire attention aux détails, et ne livre une attention accrue ni aux mots de l'énoncé, ni aux raisons de parler, ni aux effets précis de son intervention. Les locuteurs dans les contextes libres ne sont pas conscients de manière explicite des contraintes normatives affectant leur propos; par conséquent, la réalité des analyses décompositionnelles complètes du sens, qui résument ces contraintes, est moindre, et l'observation du principe REOCS est moins probable.

Les contextes propices au discours libre comprennent les conversations informelles dans lesquelles la face du locuteur n'est pas en jeu, les conversations dans lesquelles d'autres paramètres (l'affect, la connotation, les fonctions phatiques de la parole ...) priment sur le strict contenu sémantique, et les conversations sur des thèmes que le locuteur maîtrise, pouvant donc en parler facilement. Les énoncés émis dans des contextes libres présentent souvent des écarts importants par rapport aux normes grammaticales; de pareille façon, les enchaînements d'inférence dans les contextes libres peuvent bien apparaître aléatoires et peu logiques du point de vue normatif. Dans les contextes libres, le principe REOCS va moins s'appliquer, et les participants ne vérifient pas leur intercompréhension commune.

\subsection{Réalité psychologique des définitions}

On a affirmé plus haut que les énoncés ne sont pas toujours fondées sur des représentations complètes et définitionnelles observant le principe REOCS. Ce principe, on vient de le dire, s'applique le plus souvent dans le discours planifié. Dans ce genre de discours, les locuteurs emploient bel et bien des définitions et d'autres représentations explicites pour planifier et réglementer leurs énoncés - et cela de manière tout à fait délibérée et intentionnelle. Plus le locuteur souhaite que ses énoncés soient efficaces, plus il va planifier son tour conversationnel, et plus il fera attention aux divers aspects du 
référent, y accordant les expressions référentielles qu'il choisit. Souvent, cette planification ne sera pas consciente; le locuteur ne se rendra pas compte des décisions sous-conscientes qui précèdent sa contribution conversationnelle, tout en s'apercevant qu'il parle lentement ou délibérément. Pourtant, il arrive parfois que le locuteur apporte ce processus de planification au niveau de la conscience en le rendant verbal: pour évaluer les choix lexicaux différents possibles, le locuteur peut, de manière consciente, faire appel aux paraphrases verbales explicites (voir Prinz 2002 : 150). Dit autrement, le locuteur peut représenter des définitions verbales des choix lexicaux possibles. Cette remémoration des définitions permettra au locuteur d'anticiper les effets probables des divers choix lexicaux devant lui. De la même façon, le locuteur peut verbaliser une définition pour se sortir d'une panne conversationnelle occasionnée par un échec de compréhension de la part de l'auditeur.

Examinons un exemple. Soit deux cuisiniers débutants, dont l'un, $\mathrm{A}$, se met à apprendre à l'autre, $\mathrm{B}$, la bonne manière de préparer des meringues à la française, recette qu'il vient lui-même juste de maitriser. En essayant de déterminer la description correcte du geste requis pour mélanger les blancs d'oeuf et le sucre, A pourrait hésiter entre l'instruction fouetter et remuer, et s'appuyer sur des paraphrases définitionnelles conscientes, en en visualisant chaque composant, pour lui permettre d'analyser les situations décrites par chaque verbe. En réfléchissant sur les alternatives, A pourrait associer fouetter avec la paraphrase « faire monter avec des mouvements courts et rapides du poignet pour rendre mousseux » et remuer avec « tourner sans arrêt pour mélanger ». A n'est évidemment pas obligé d'employer de telles paraphrases pour décider quel est le bon verbe à choisir. Il existe sans doute d'autres moyens de trancher. Néanmoins, l'appel à de telles paraphrases est un moyen possible, surtout dans les cas où, comme dans l'illustration présente, le locuteur aborde un thème conversationnel nouveau en tant que débutant, ne disposant pas encore d'une gamme de choix lexicaux bien établie, ou encore plus dans un contexte abstrait où les significations ne peuvent pas être visualisées.

L'appel à une paraphrase définitionnelle peut aussi se faire dans le sens contraire. Pour ce qui est de nos cuisiniers, il se peut que A, ayant dans l'esprit l'instruction «faire monter avec des mouvements courts et rapides du poignet pour rendre mousseux ", s'aperçoit que le verbe fouetter communique cette message de manière plus économique. Dans un tel cas, le locuteur associe sous-consciemment un lexème (fouetter) avec une paraphrase verbale.

On peut également rendre explicites les paraphrases verbales lorsqu'il s'agit de réparer une panne. Si B ne répond pas de façon appropriée à l'instruction fouetter, A peut verbaliser la structure définitionnelle pour réparer cette panne de compréhension. Les définitions verbalisées servent souvent de réparer les pannes conversationnelles de cette façon.

De telles paraphrases sont des définitions adaptées à des contextes précises: elles décomposent une signification dans des unités plus élémentaires, étiquetant ces unités avec des mots de la même langue, pour tirer l'attention sur des aspects précis du référent.

Pourtant, il existe une différence importante entre les deux situations que l'on vient d'évoquer (verbalisation d'une définition par le locuteur pour éclaircir son propos devant l'auditeur; remémoration privée par le locuteur d'une définition pendant la formulation mentale de l'énoncé). Les contraintes temporelles qui pèsent sur la performance en ligne limitent la possibilité d'élaborer des définitions mentales pendant la préparation de la 
parole. En revanche, un délai plus important existe pour la verbalisation d'une définition. Un locuteur peut prendre tout son temps pour articuler la définition d'un mot devant le locuteur, mais dispose d'un délai plus court lorsqu'il prépare intérieurement sa prochaine contribution conversationnelle. Les définitions construites par les locuteurs pendant la planification de l'énoncé comprendront donc une moindre quantité de composants que leurs équivalents élaborés hors-ligne. Ils ne sont pas complets, parce qu'ils ne ciblent que les aspects pertinents de la situation à laquelle l'expression fait référence, déterminés par l'attention et les buts conversationnels du locuteur, ainsi que par le contexte de l'énoncé.

Les spéculations qui viennent d'être présentées permettent de clarifier la manière dont le contexte peut influer sur le statut psychologique des analyses décompositionnelles de sens telles que l'on avance en sémantique. Si les spéculations ici sont bonnes, de telles analyses deviennent psychologiquement réelles au sein de deux catégories de contexte planifié. Premièrement, les locuteurs peuvent parfois rappeler des définitions verbales minimales pendant la préparation mentale de leurs énoncés. Deuxièmement, les locuteurs peuvent verbaliser ces définitions pour réparer une panne de compréhension de la part de l'auditeur.

\subsection{Passage discours planifié-discours libre}

Les contextes planifiés confèrent donc un certain degré de réalité psychologique aux représentations complètes décompositionnelles. Au fur et à mesure que le degré de planification diminue, deux phénomènes se produisent. En premier, la spontanéité grandissante dispense le locuteur du besoin de représenter le référent de manière précise, et les représentations sous-tendant le discours deviennent de plus en plus minimales, holistes et préprogrammées. L' «entrenchment ", les effets de gestalt, la routinisation, entre autres facteurs, rendent moins nécessaires des structures complètes et élaborées comme les supports des énoncés (cf. Langacker 1987; Givón 1995; Verschueren 1999 ; Bybee 2006). Les locuteurs font moins attention à leurs choix lexicaux: le locuteur demande à l'auditeur de passer la balle au lieu de la raquette parce que, par exemple, le lexème balle était mentionné dans une phrase précédente (Szmrecsanyi 2005; Harley and MacAndrew 2001). L'interprétation que fait l'auditeur dépend moins du contenu normatif de l'énoncé, et plus de ses attentes quant à la signification probable dans le contexte (cf. Hopper 1998 ; Sanford 2002). L'auditeur passe la batte non pas parce que la batte satisfait le concept évoqué par le mot raquette, mais parce qu'il voit que le locuteur désire jouer au ping-pong, et anticipe qu'il en aura besoin. De tels effets font que le traitement sémantique est souvent très peu profond, et démontrent que la référence réussie peut se produire sans représentation complète du contenu sémantique.

Deuxièmement, plus le contexte devient libre, plus le contenu connotatif et expressif vient au premier plan (Potts 2007 ; Ochs et Schieffelin 1989 ; cf. Blakemore 2011). Plus le locuteur diminue son attention aux contraintes normatives explicites, plus la dimension affective/expressive de sens détermine l'enchaînement du discours et, dans un moindre degré, la référence (Riemer 2013). Moins je me concentre sur les contraintes normatives, plus libres deviennent les inférences que je peux tirer. Comme on le remarque souvent, les énoncés peuvent souvent résulter des réactions émotionnelles avant des réactions raisonnées. Je peux traiter quelqu'un de mon ami non pas pour attribuer à notre relation les caractéristiques reconnues socialement de l'amitié, mais purement pour exprimer un 
affect positif à son égard. Dans un tel cas, le contenu descriptif peut être neutralisé par le sens expressif.

\section{Conclusion}

Dans la première partie de l'article, on a remis en question l'idée traditionnelle selon laquelle le contenu sémantique littéral ne comprend que l'information minimale qui est invariable à travers des contextes différents. On a discuté de la manière dont le principe REOCS empêche l'analyse sémantique d'attribuer un rôle non-supplétif au contexte dans la production du sens. Ensuite, j'ai suggéré deux effets contextuels sur la représentation $\mathrm{du}$ contenu sémantique, riches en conséquences pour les modèles en sémantique linguistique. Dans le premier effet, l'auditeur n'a même pas besoin de représenter le sens littéral complet d'une expression pour manifester une " compréhension " adéquate, parce que le contexte fournit déjà l'information nécessaire pour réussir une interaction linguistique. Par conséquent, l'hypothèse selon laquelle les représentations sémantiques en temps réél de traitement sont souvent sous-minimales paraît raisonnable. Deuxièmement, on a abordé la contribution du contexte de discours à la représentation sémantique, en particulier les effets du degré de l'attention du locuteur sur la nature des représentations sémantiques en temps réél de traitement, en faisant la différence entre les contextes planifiés et libres. On a soutenu l'idée selon laquelle le passage depuis un contexte libre vers un contexte planifié entraîne des modifications aux représentations sémantiques impliquées dans la performance linguistique.

Dans la deuxième partie de cet article j'ai soutenu l'idée selon laquelle une sorte de contexte interne, à savoir le degré de planification, influe sur les significations communiquées. Si c'est le cas, cela enlève à la sémantique l'exemption surprenante de l'influence de facteurs contextuels qui affectent, on l'a démontré souvent, les autres niveaux de la structure linguistique. Si ces autres dimensions de la performance linguistique sont concernées par le degré d'attention consciente du locuteur, il n'est aucunement surprenant de découvrir que l'on constate également les différences sémantiques selon que le locuteur prête un degré d'attention élevé ou amoindri à ses paroles.

En somme, les possibilités spéculatives évoquées dans l'article présent soulèvent des questions sur le statut empirique des représentations riches typiquement supposées dans la sémantique linguistique, et augmente le répertoire des interactions entre contexte et sens qu'il faut prendre en compte dans le but de développer une théorie naturalise de la compétence sémantique.

\section{BIBLIOGRAPHIE}

Anderson, J. R. 1983. The architecture of cognition. Cambridge, MA : Harvard University Press.

Anderson, M. 2003. «Embodied cognition : a field guide ». Artificial Intelligence 149 : 91-130. 
Barsalou, L. 1999. «Perceptual symbol systems ». Behavioral and Brain Sciences 22 : 577-660

Barsalou, L. 2008. « Grounded cognition ». Annual Review of Psychology 59 : 617-645.

Barton, Stephen B and Sanford, Anthony J. 1993. « A case study of anomaly detection : Shallow semantic processing and cohesion establishment ». Memory and Cognition 21 : 477-487.

Beer, Randall D. 2000. « Dynamical approaches to cognitive science ». Trends in Cognitive Sciences $4:$ : 91-99.

Besnier, N. 1990. « Language and affect ». Annual Review of Anthropology 19 : 419-451.

Binder, J., Desai, R.H., 2011. The neurobiology of semantic memory. Trends in Cognitive Sciences 15 : 527-536.

Blakemore, Diane 2011. On the descriptive ineffability of expressive meaning. Journal of Pragmatics 43 : 3537-3550

Breheny, Richard 2002. The Current State of (Radical) Pragmatics in the Cognitive Sciences Mind \& Language $17: 169-187$.

Brooks, R. 1991. Intelligence without representation. Artificial Intelligence 47 139-159

Bybee, J. 2006. From Usage To Grammar : The Mind's Response To Repetition. Language 82 : 711733.

Caffi, C. and Janney, R., 1994b Toward a pragmatics of emotive communication. Journal of Pragamatics 22, 325-373.

Cann, Ronnie, Ruth Kempson and Eleni Gregoromichelaki. Semantics. Cambridge : CUP.

Clark, Andy. 1997. Being There. Putting Brain, Body and World Together Again. Cambridge, MA : MIT Press.

Clark, Andy. 1998. Embodied, situated, and distributed cognition. In A Companion to Cognitive Science, William Bechtel and George Graham (eds.), 506-518. Malden/Oxford : Blackwell.

Clark, E.V.. 2009. Lexical meaning. In Bavin, E.L., ed. The Cambridge Handbook of Child Language 283300.

Coupland, Nikolas. 2007. Style. Language Variation and identity. CUP.

Daneman, Meredyth, Lennertz, Tracy and Hannon, Brenda. 2006. Shallow semantic processing of text : Evidence from eye movements, Language and Cognitive Processes 22 : 83- 105

de Fornel, Michel \& Louis Quéré (eds) 1999. La logique des situations. Nouveaux regards sur l'écologie des activités sociales. Paris : Editions de l'école des hautes études en sciences sociales.

Dreyfus, H. 1991 Being in the World. A commentary on Heidegger's Being and Time, division I. Cambridge, MA : MIT Press.

Eckert, P. 2000 : Linguistic variation as social practice. Oxford : Blackwell.

Erickson, T. A., \& Mattson, M. E. (1981). From words to meaning : A semantic illusion. Journal of Verbal Learning \& Verbal Behavior 20 : 540-552.

Frisson, S. (2009), Semantic Underspecification in Language Processing. Language and Linguistics Compass $3: 111-127$.

Gadamer, H-G. 1960. Truth and Method. London : Sheed and Ward.

Geeraerts, Dirk 2010 Theories of Lexical Semantics. Oxford : Oxford University Press 
Gibbs, Raymond W. Jr. 2002. A new look at literal meaning in understanding what is said and implicated, Journal of Pragmatics 34 : 457-486.

Gibson, James G. 1986 [1979]. The ecological approach to visual perception. Hillsdale : Erlbaum Giora, Rachel. 2012. The psychology of utterance processing. In Allan, Keith and Jaszczolt, Kasia M. The Cambridge Handbook of Pragmatics. Cambridge : CUP, 151-167

Givón, T. 1995. Functionalism and Grammar. Amsterdam : Benjamins.

Givón, T. 2001 Toward a neuro-cognitive interpretation of 'context'. Pragmatics \& Cognition 9 : 175-201.

Glenberg AM. 1997. What memory is for. Behavioral and Brain Sciences. $20: 1-55$

Harley Trevor A. and Siobhan B. G. MacAndrew. 2001. Constraints Upon Word Substitution Speech Errors. Journal of Psycholinguistic Research, 30 : 395-418.

Hopper, Paul J. 1998. Emergent grammar. In Tomasello, Michael J. (ed) The new psychology of language. Mahwah : Erlbaum. Vol. I, 155-175

Kirsh, D. 1995. The intelligent use of space. Artificial Intelligence 73 : 31-68.

Labov 1972 Sociolinguistic patterns. Philadelphia : University of Pennsylvania Press.

Langacker, R. 1987. Foundations of Cognitive Grammar. Chicago.

Merleau-Ponty, M. 1946. Phénoménologie de la perception. Paris : Gallimard.

Nyckees, Vincent 1998. La sémantique. Paris : Belin.

Ochs, E. 1979 Planned and unplanned discourse, in T. Givón ed. Syntax and semantics 12 : discourse and syntax. New York : Academic.

Ochs, Eleanor and Schieffelin, Bambi 1989. Language has a heart. Text 9:7-25.

Pinker, S. and Prince, A. (1988) On language and connectionism : analysis of a parallel distributed processing model of language acquisition. Cognition 28, 73-193.

Port, R. 2010. Rich memory and distributed phonology. Language Sciences 32 : 43-55.

Port, R. and Leary, A. 2005. Against formal phonology. Language 81 : 927-964.

Potts, C. 2007. The expressive dimension. Theoretical Linguistics $33: 165-198$

Prinz, J. 2002. Furnishing the mind : concepts and their perceptual basis. Cambridge MA : MIT.

Pustejovsky, J. 1998. The semantics of lexical underspecification. Folia Linguistica 32 : 323-347.

Rastier, François 2001. Sémantique et recherches cognitives. 2ed. Paris : PUF

Recanati, François 2012. Pragmatic Enrichment. In Russell, G. and D. Graff Fara (eds) Routledge Companion to Philosophy of Language, pp.67-78.

Riemer, Nick 2013. Conceptualist semantics : explanatory power, scope and uniqueness. Language Sciences $35: 1-19$.

Sanford, A.J. 2002. Context, attention and depth of focussing during interpretation. Mind and language 17 : 188-206.

Schilling-Estes Natalie. 2007. Sociolinguistic fieldwork. In Robert Bayley and Ceil Lucas (eds) Sociolinguistic theory. Theories, methods, and applications. Cambridge : CUP, 165-189.

Smith, E. E., Shoben, E. J., \& Rips, L. J. 1974. Structure and process in semantic memory : A featural model for semantic decision. Psychological Review, 81, 214-241 
Sperber, Dan and Wilson, Deidre. 1995. Relevance. Communication and cognition. 2ed. Oxford : Blackwell.

Szmrecsanyi, B. 2005. Language users as creatures of habit : a corpus-based analysis of persistence in spoken English. Corpus Linguistics and Linguistic Theory 1 : 113-149.

Van Gelder, T. 1998 : The dynamical hypothesis in Cognitive Science. Behavioral And Brain Sciences $21: 615-665$

Van Oostendorp, H., \& De Mul, S. 1990. Moses beats Adam : A semantic relatedness effect on a semantic illusion. Acta Psychologica 74 : 35-46.

Van Oostendorp, H., \& Kok, I. 1990. Failing to notice errors in sentences. Language \& Cognitive Processes $5: 105-113$

Verschueren, J. 1999. Understanding Pragmatics. London : Arnold.

Wierzbicka, A. 1996. Semantics. Primes and Universals. Oxford : OUP.

\section{RÉSUMÉS}

Le présent article aborde deux effets de contexte, peu étudiés, sur la représentation du contenu sémantique, en considérant les implications que peuvent avoir ces effets pour la modélisation sémantique. Dans le cas de la sous-minimalité sémantique, le contexte rend même le contenu sémantique minimal (définitionnel) redondant pour ce qui est du traitement cognitif réussi d'une expression(sa compréhension). Deuxièmement, on aborde la manière dont le contexte de discours influe sur la nature des représentations sémantiques en ligne, en établissant une différence entre le discours planifié et le discours libre en tant que deux contextes différents pour ce qui est de la nature des représentations sémantiques sous-jacentes. En abordant ces deux questions, l'article remet en question l'importance des représentations riches que l'on suppose habituellement dans la sémantique linguistique, et augmente le répertoire des interactions senscontexte dont il faut rendre compte dans une théorie naturaliste de la compétence sémantique.

Two little studied effects of context on the representation of semantic content are discussed, and their implications for linguistic semantic models generally are considered. In cases of semantic subminimality, the hearer does not have to represent an expression's full literal meaning in order to achieve contextually appropriate understanding, since the context already supplies the information necessary for successful linguistic interaction. Second, there are persuasive, though speculative, reasons to believe that the nature of semantic representation varies along with the discourse context, particularly as concerns the contrast between planned and free contexts of utterance. These two contextual effects raise questions about the empirical status of the rich representation typically assumed in linguistic semantics, and supplement the inventory of context-meaning interactions that arguably need to be considered in attempts to develop a naturalistic theory of semantic competence.

\section{INDEX}

Mots-clés : représentation sémantique, sens littéral, dépendance de contexte, interprétation pragmatique, attention, traitement sémantique, définitions

Keywords : semantic representation, literal meaning, context-dependence, pragmatic interpretation, attention, semantic processing, definitions 


\section{AUTEUR}

\section{NICK RIEMER}

University of Sydney, Australie et Laboratoire d'histoire des théories linguistiques, CNRS, Université Paris-Diderot 